\title{
Allergologie kompakt und kompetent zusammengefasst
}

— Im Oktober 2016 kam das Werk Allergologie kompakt heraus - ein Buch, das im Verlauf mehrerer Veranstaltungen der früheren Allergie-Akademie, jetzt Allergologie Kompakt, der Deutschen Gesellschaft für Allergologie und klinische Immunologie (DGAKI) aus den Beiträgen der interdisziplinär zusammengesetzten Referenten hervorgegangen ist. Die 26 Kapitel stehen jeweils für sich, bauen insgesamt aber schlüssig aufeinander auf und umfassen alle relevanten Themen des allergologischen Curriculum.

\section{Die ganze Welt der Allergologie}

Die Darstellungen reichen von der Geschichte der Allergie, der neuen Nomenklatur, die durch die molekulare Allergologie geprägt wurde, den immunologischen Grundlagen, einem umfassenden Kapitel zu den Einzelallergenen, den Symptomen an verschiedenen Organsystemen und leiten zur Diagnostik und Therapie sowie Prävention über. Wenn jeweils unter den einzelnen Organsystemen nochmals auf die Diagnostik eingegangen wird, so hat der Leser zuvor bereits einen kompakten Überblick über die verschiedenen auf dem Markt befindlichen Verfahren bekommen und folgt dankbar der kritischen Beschreibung im jeweils neuen Kapitel. Besonders wertvoll sind Übersichten bezüglich der Differenzialdiagnosen.

\section{Rund um die Therapie}

Die Therapie wird ausführlich erklärt, wobei der Pharmakotherapie im Sinne der
„Therapeutischen Prinzipien“ ein gut strukturierter Überblick gewidmet wird. Sowohl die symptomatische als auch die spezifische Immuntherapie (SIT) sind umfassend und teilweise mit anschaulichen Beispielen beschrieben, wobei insbesondere auch die in der Praxis immer wieder auftretenden Grenzsituationen geschildert werden. Bezüglich der Auswahl der Patienten für eine SIT wird - kurz zusammengefasst - die Diagnostik und die daraus resultierende Therapieentscheidung dargestellt. Die Frage, wer wann welche Therapie erhält, wird kompetent und kompakt beschrieben vor dem Hintergrund der aktuellen Studienlage.

\section{Praxisnah und interdisziplinär}

Zum Ende des Buchs wird die berufliche Auslösung allergischer Erkrankungen interdisziplinär dargestellt. Übergreifenden Phänomenen wie dem Pruritus und den psychologischen Aspekten ist jeweils ein Extrakapitel gewidmet. Als weitere Besonderheit beinhaltet Allergologie kompakt Multiple-Choice-Fragen zu den abgehandelten Kapiteln (inklusive Auflösung zur Selbstkontrolle).

Das Buch "Allergologie kompakt" führt elegant geschrieben durch alle praktisch relevanten Aspekte der interdisziplinären Allergologie. So wird sich jeder, der allergische Patienten zu versorgen hat, gleich welcher Fachdisziplin er angehört, übersichtlich und rasch auf dem neuesten Stand der klinischen Forschung und der Leitlinien informiert fühlen.

\footnotetext{
Allergologie Kompakt - die Veranstaltung

Die DGAKI-Veranstaltung „Allergologie Kompakt“, aus denen das gleichnamige Buch hervorgegangen ist, findet Anfang 2017 in Hannover statt.

Termin

26.-28. Januar 2017

Tagungsort

Courtyard by Marriott Hannover Maschsee

Teilnahmegebühren

DGAKI-Mitglieder: $250 €$

Nichtmitglieder: $450 €$

\section{Programmkomitee}

Prof. Dr. Ulrike Raap, Hannover Prof. Dr. Ulf Darsow, München

Anmeldung und Reisestipendien

DGAKI-Geschäftsstelle

Robert-Koch-Platz 7, 10115 Berlin

Tel.: (030) 28047100 / Fax: (030) 28047101

E-Mail: info@dgaki.de

Internet: www.allergieakademie.de
}

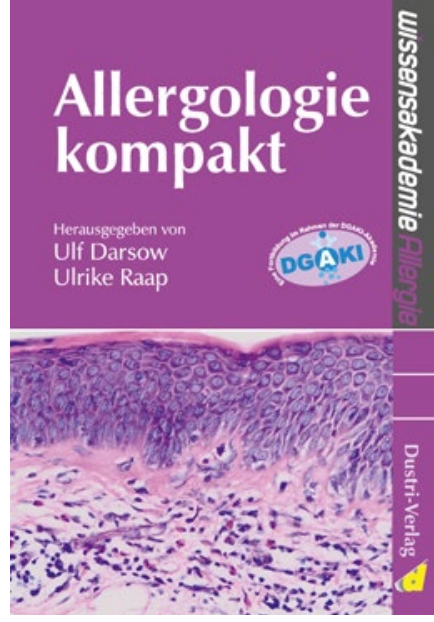

Ulf Darsow, Ulrike Raap

Allergologie kompakt

Wissensakademie Allergie

1. Auflage

Dustri Verlag Verlag

Oberhaching 2016

ISBN 978-3-87185-483-5

$118 €$

\section{Nicht nur für Ärzte in Weiterbildung}

Das Buch wird sowohl diejenigen Ärzte ansprechen, die sich noch in der allergologischen Weiterbildung befinden und ein Werk zur Prüfungsvorbereitung suchen, als auch die bereits erfahrenen Allergologen, die sich gut in den geschilderten Erfahrungswerten (und "Tricks") der Autoren wiederfinden.

Das Werk ist sehr praxisnah, Abbildungen und Tabellen dienen erfolgreich der Vermittlung der relevanten Fakten und strukturieren die Informationen sinnvoll. Einige Kapitel bieten zusätzlich eine kurze Zusammenfassung am Schluss oder Absätze mit der Überschrift „Praktisches Vorgehen".

Bei allen Beiträgen wird die jahrelange Erfahrung in der klinisch-allergologischen Arbeit sichtbar, die Freude am Thema und an dem wissenschaftlichen Potenzial, das die Allergologie bietet.

Das Buch Allergologie kompakt ist sowohl kompakt als auch kompetent und dabei so viel mehr als ein „schneller Überblick“.

Uta Jappe 